\title{
Variabilidade na sensibilidade à heparina de reagentes do TTPA em plasmasheparinizado in vitro e de pacientes heparinizados
}

\section{Variability in heparin sensitivity of TTPA reagents in heparinized plasma in vitro and patients receiving heparin}

\author{
Fernanda Daniela Serralvo'; Jacinta Ludovico Zamboti²; Maria Emilia Favero³; José \\ Wander Bregano ${ }^{4}$
}

\begin{abstract}
Resumo
Introdução: No final da década de 80 e início dos anos 90 vários estudos demonstraram a falta de padronização e a variabilidade nos resultados do TTPA devido a diferentes sensibilidades à heparina dos reagentes empregados para sua determinação.

Objetivo: Avaliar a sensibilidade à heparina de reagentes utilizados para a determinação do TTPA em amostras de plasmas heparinizadas in vitro e de pacientes em uso de heparina não fracionada (HNF).

Material e Métodos: Para este estudo foi utilizado um pool de plasma heparinizado, com concentrações de 0,1 até 1,0 unidade de heparina/mL, 29 pacientes em uso de HNF e 8 kits de reagentes para a determinação do TTPA.

Resultados e Discussão: Com os plasmas heparinizados in vitro os resultados com o reagente da Actin ${ }^{\circledR}$ foram estatísticamente diferente dos da Labtest ${ }^{\circ}$, da Human ${ }^{\circledR}$ e da Clot ${ }^{\circledR}$. O melhor coeficiente de correlação, esultado do TTPA versus concentração de HNF, foi observado com o reagente da Stago $\mathbb{R}(\mathrm{R}=0,9919)$. Quando se empregou os plasmas de pacientes em uso de HNF os resultados do Actin ${ }^{\circledR}$ e do Actin FSL ${ }^{\circledR}$ foram estatisticamente diferentes dos da Clot ${ }^{\circledR}$.

Conclusão: Diferenças estatisticamente significativas, nos valores de TTPA, ainda são observadas, tanto em plasmas de pacientes em uso de HNF como em plasmas heparinizados in vitro de acordo com o reagente utilizado.
\end{abstract}

Palavras-chave: Tempo de tromboplastina parcial ativado. Heparina. Anticoagulantes. Monitoramento. Sensibilidade.

\footnotetext{
Abstract

Introduction: At the end of the 1980s and at the beginning of the 1990s, several studies showed lack of standardization and variability in APPT results due to the different sensibilities to heparin in reagents used for its determination.

Objective: To evaluate the sensibility to heparin in the different reagents used to determine APPT in samples of heparinized plasma in vitro and in patients using non-fractioned heparin (NFH).

Material and Methods: This study was performed with a pool of heparinized plasma with concentrations from 0.1 to 1.0 unit heparin/mL, 29 patients using NFH and 8 reagent kits for TTPA determination.

Results and Discussion: Using heparinized plasma in vitro, there was a statistically significant difference with Actin $\AA$ reagent in relation to Labtest $\AA$, Human ${ }^{\circledR}$ and $\operatorname{Clot} \AA$ reagents. The best correlation coefficient, a

${ }^{1}$ Graduação em Farmácia - Residente em Análises Clinicas - Departamento de Análises Clínicas e Toxicológicas - PAC/CCS/ Universidade Estadual de Londrina, Brasil.

${ }^{2}$ Professora Doutora do Departamento de Estatística do CCE/Universidade Estadual de Londrina, Brasil.

${ }^{3}$ Professora Mestre (Mestrado em Farmácia/Análises Clínicas) do Departamento de Análises Clínicas e Toxicológicas do CCS/ Universidade Estadual de Londrina, Brasil.

${ }^{4}$ Professor Doutor (Doutorado em Ciências da Saúde) do Departamento de Patologia Análises Clínicas e Toxicológicas do CCS/ Universidade Estadual de Londrina, Brasil.
} 
result of the APTT versus UFH concentration was observed with reagent from Stago $\mathbb{R}(\mathrm{R}=0.9919)$. When we used the plasma from patients using UFH, the results of Actin ${ }^{\circledR}$ and Actin FSL ${ }^{\circledR}$ were statistically different from the Clot ${ }^{\circledR}$.

Keywords: Activated partial thromboplastin time. Heparin. Anticoagulant. Monitoring. Variability.

\section{Introdução}

O Tempo de Tromboplastina Parcial Ativado (TTPA) é um teste amplamente utilizado para detectar a deficiência de fatores na via intrínseca e comum do sistema de coagulação e a presença de inibidores, tais como o anticoagulante lúpico. $\mathrm{O}$ TTPA também é utilizado para monitorar a terapia heparínica, o prolongamento do tempo de coagulação é proporcional a dose deste anticoagulante (LIPPI; FAVALORO, 2008).

A heparina é um potente anticoagulante utilizado na profilaxia e tratamento de doenças como a trombose venosa profunda, o embolismo pulmonar, $\mathrm{o}$ infarto agudo do miocárdio e em certos procedimentos como a hemodiálise. $\mathrm{O}$ efeito anticoagulante in vivo depende de vários fatores como o tipo de heparina administrada, o tipo de medicamento administrado concomitantemente e o metabolismodopaciente, desta maneira é importante monitorar laboratorialmente a terapia com heparina pela determinação do TTPA para adequar a dose de heparina ao efeito anticoagulante desejado (HAMERSCHLAK; ROSENFELD, 1996).

Nas décadas de 80 e 90 vários estudos demonstraram a falta de padronização dos reagentes empregados para a determinação TTPA e a variabilidade na sensibilidade à heparina não fracionada (HNF). Esta variabilidade na sensibilidade se deve, principalmente, pela constituição do reagente, portanto estudos deveriam ser realizados para padronizar os resultados deste exame. Também foi demonstrado que esta variabilidade pode interferir na prescrição deste anticoagulante podendo levar o paciente a um estado de anticoagulação indesejado tendo um risco aumentado de hemorragia ou trombose (BJORNSSON; NASH, 1986; KITCHEN et al., 1996; KITCHEN et al., 1999, POLLER, THOMSON, TABENER, 1989).
O objetivo principal deste estudo foi avaliar a variabilidade nos resultados do TTPA utilizando oito kits comerciais e empregando plasmas heparinizados in vitro e plasmas de pacientes em uso de HNF.

\section{Materiais e Métodos}

\section{Pacientes e Obtenção das Amostras}

Para este estudo foram selecionados aleatoriamente 29 pacientes internados no Hospital Universitário da Universidade Estadual de Londrina, que faziam o uso de HNF por via endovenosa ou subcutânea. $\mathrm{O}$ sangue foi colhido em tubos à vácuo, marca Becton Dickinson, contendo citrato de sódio 3,2\% como anticoagulante. As amostras de plasma foram congeladas em nitrogênio líquido e posteriormente foram mantidas em congelador a $-70^{\circ} \mathrm{C}$ até a realização dos testes. As amostras hemolisadas, lipêmicas, ictéricas e/ou que não apresentaram a proporção sangue/anticoagulante 9:1 foram excluídas do estudo. Este estudo foi aprovado pelo Comitê de Bioética e Ética em Pesquisa da Irmandade Santa Casa de Londrina - BIOISCAL e os termos de consentimento, para a participação no estudo, foram obtidos de cada paciente.

\section{Preparação do pool de Plasma}

O pool de plasma empregado para o cálculo da relação ( $\mathrm{R}$ = TTPA paciente/TTPA pool) e para a realização da curva de plasmas heparinizados foi preparado pela mistura de 25 amostras de plasma de doadores de sangue atendidos no Hemocentro do Hospital Universitário. 
Reagentes utilizados para realização do TTPA

Foram utilizados oito kits comerciais para a determinação do TTPA: Actin (ACTIN) e Actin FSL (ACTIN FS) da marca Dade-Behring®, STA PTT

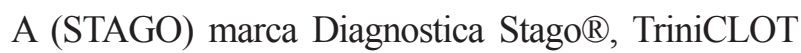

aPTTS(TRINITYS) e TriniCLOTaPTTHS(TRINITY HS) marca Trinity Biotech ${ }^{\circledR}$, APTT Hemostasis (LABTEST) marca Labtest ${ }^{\circ}$, TTPA CLOT (CLOT) marca Bios Diagnostica ${ }^{\circledR}$ e HEMOSTAT aPTT-EL (HUMAN) marca Human do Brasil®. A composição dos reagentes utilizados está descrita na Tabela 1.

Tabela 1 - Composição dos reagentes utilizados para a determinação do Tempo de Tromboplastina Parcial Ativado (TTPA)

\begin{tabular}{|c|c|c|}
\hline MARCA & PRODUTO & COMPOSIÇÃO* \\
\hline $\begin{array}{c}\text { BIOS } \\
\text { DIAGNÓSTICA }^{\circledR}\end{array}$ & TTPA CLOT & $\begin{array}{l}\text { Composto de extrato de cérebro de coelho, ácido elágico tampão e } \\
\text { conservante }\end{array}$ \\
\hline DADE-BEHRING ${ }^{\circledR}$ & $\operatorname{Actin}^{\circledR}$ & $\begin{array}{c}\text { Ácido elágico para a ativação dos fatores XII e XI, extrato de } \\
\text { cérebro de coelho, um fosfolipídio como um substituto de um fator } 3 \\
\text { plaquetário e íons de cálcio }(0,02 \mathrm{~mol} / \mathrm{L})\end{array}$ \\
\hline DADE-BEHRING ${ }^{\circledR}$ & $\operatorname{Actin}^{\circledR}$ FSL & $\begin{array}{l}\text { Factor Sensível (FS) contém ácido elágico para a ativação dos fatores } \\
\text { XII e XI, bem como um fosfolipídeo de soja como substituto de um } \\
\text { fator } 3 \text { plaquetário. Este sistema reagente é também complementado } \\
\text { com íons de cálcio }(0,02 \mathrm{~mol} / \mathrm{L}) \text {. }\end{array}$ \\
\hline $\begin{array}{l}\text { DIAGNOSTICA } \\
\text { STAGO }^{\circledR}\end{array}$ & STA PTT A ${ }^{(5)}$ & $\begin{array}{l}\text { Reagente liofilizado contendo um substituto plaquetário (cefalina) } \\
\text { extraído de tecido cerebral de coelho e um ativador de partículas } \\
\text { (silício) em meio tamponado. Cloreto de Cálcio } 0,025 \mathrm{M} \text {. }\end{array}$ \\
\hline $\begin{array}{l}\text { HUMAN DO } \\
\text { BRASIL }^{\circledR}\end{array}$ & $\begin{array}{l}\text { HEMOSTAT aPTT- } \\
\text { EL }\end{array}$ & $\begin{array}{l}\text { Extrato cloroformizado de cérebro de coelho (fosfolipede) } 0,007 \% \text {, } \\
\text { ácido elágico } 0,0037 \% \text {. Cloreto de cálcio } 0,02 \mathrm{~mol} / \mathrm{L} \text {. Azida } 0,1 \% \text {. }\end{array}$ \\
\hline LABTEST $^{\circledR}$ & APTT Hemostasis & $\begin{array}{l}\text { Contém ácido elágico }>99 \mathrm{mmol} / \mathrm{L} \text {, fosfolipede de cérebro de coelho } \\
>0,007 \% \text {, fenol } 320 \mathrm{mmol} / \mathrm{L} \text {, albumina bovina } 0,005 \% \text {, estabilizadores } \\
\text { e preservativo. Cloreto de cálcio } 20 \mathrm{mmol} / \mathrm{L} \text { e azida sódica }<0,095 \% \text {. }\end{array}$ \\
\hline $\begin{array}{c}\text { TRINITY } \\
\text { BIOTECH }^{\circledR}\end{array}$ & TriniCLOT aPTT HS & $\begin{array}{l}\text { Fosfolipídeos purificados (porco e galinha); contém sílica micronizada } \\
\text { (ativador), tampão, estabilizador e conservante. }\end{array}$ \\
\hline $\begin{array}{c}\text { TRINITY } \\
\text { BIOTECH }^{\circledR}\end{array}$ & TriniCLOT aPTT S & $\begin{array}{l}\text { Fosfolipídeos purificados (porco e galinha); contém sílica micronizada } \\
\text { (ativador), tampão, estabilizante e azida sódica a } 0,02 \% \text { como } \\
\text { conservante. }\end{array}$ \\
\hline
\end{tabular}

* Composição informada na bula de cada produto

Fonte: autores

\section{Curva de heparina}

A curva de heparina foi realizada a partir do pool de plasmas com adição de HNF para se obter as concentrações finais de 0,$1 ; 0,2 ; 0,3 ; 0,4 ; 0,5 ; 0,6$; 0,$7 ; 0,8 ; 0,9$ e 1,0 unidade de heparina $/ \mathrm{mL}$.

\section{Realização dos testes coagulométricos}

As amostras de plasma dos pacientes foram descongelados rapidamente em banho-maria à $37^{\circ} \mathrm{C}$. A determinação do TTPA foi realizada em equipamento automatizado de coagulação da marca Dade Behring®, modelo Behring Coagulation Timer (BCT), utilizando os reagentes citados anteriormente. Os TTPAs da curva de heparina foram realizados em duplicata. O equipamento foi programado conforme recomendação de cada fabricante.

\section{Teste de repetibilidade}

Para o teste de repetibilidade foram selecionadas duas amostras, uma com o valor dentro dos valores de referência (amostra A) e a outra com resultado alterado (amostra B) de TTPA com o reagente da Dade Behring®. Foram realizadas vinte determinações do TTPA para cada amostra com os oito reagentes selecionados. Os resultados do TTPA foram expressos em segundos e R. A partir destes resultados foram determinados a média, desvio padrão (DP) e coeficiente de variação (CV). 
Análise estatística

O Teste de Friedman foi utilizado para verificar as diferenças entre os resultados de TTPA expressos em $\mathrm{R}$ obtidos com os diferentes kits comerciais avaliados. Foi considerada significância estatística quando o $\mathrm{p}<0,05$.

\section{Resultados}

Utilizando plasmas heparinizados in vitro (curva de heparina) os resultados com o reagente Actin ${ }^{\circledR}$ foram estatísticamentes diferentes daqueles obtidos com os reagentes da Labtest ${ }^{\circledR}$, Human $\AA$ e $\operatorname{Clot} \AA$. Por sua vez, os resultados com reagente Human $\mathbb{}$ foram significativamente diferentes dos da Stago ${ }^{\circledR}$ e da Trinity $\mathrm{S} \circledast$ (Figura 1).

Figura 1 - Resultados de TTPA expressos em relação (TTPA plasma heparinizado/ TTPA pool de plasma), empregando oito kits comerciais e plasmas heparinizados in vitro. a- Actin versus Labtest, Human e Clot; b- Human versus Stago e Trinity S. Análise estatística realizada pelo Teste de Friedman, foi considerado significância estatística quando o $\mathrm{p}<0,05$

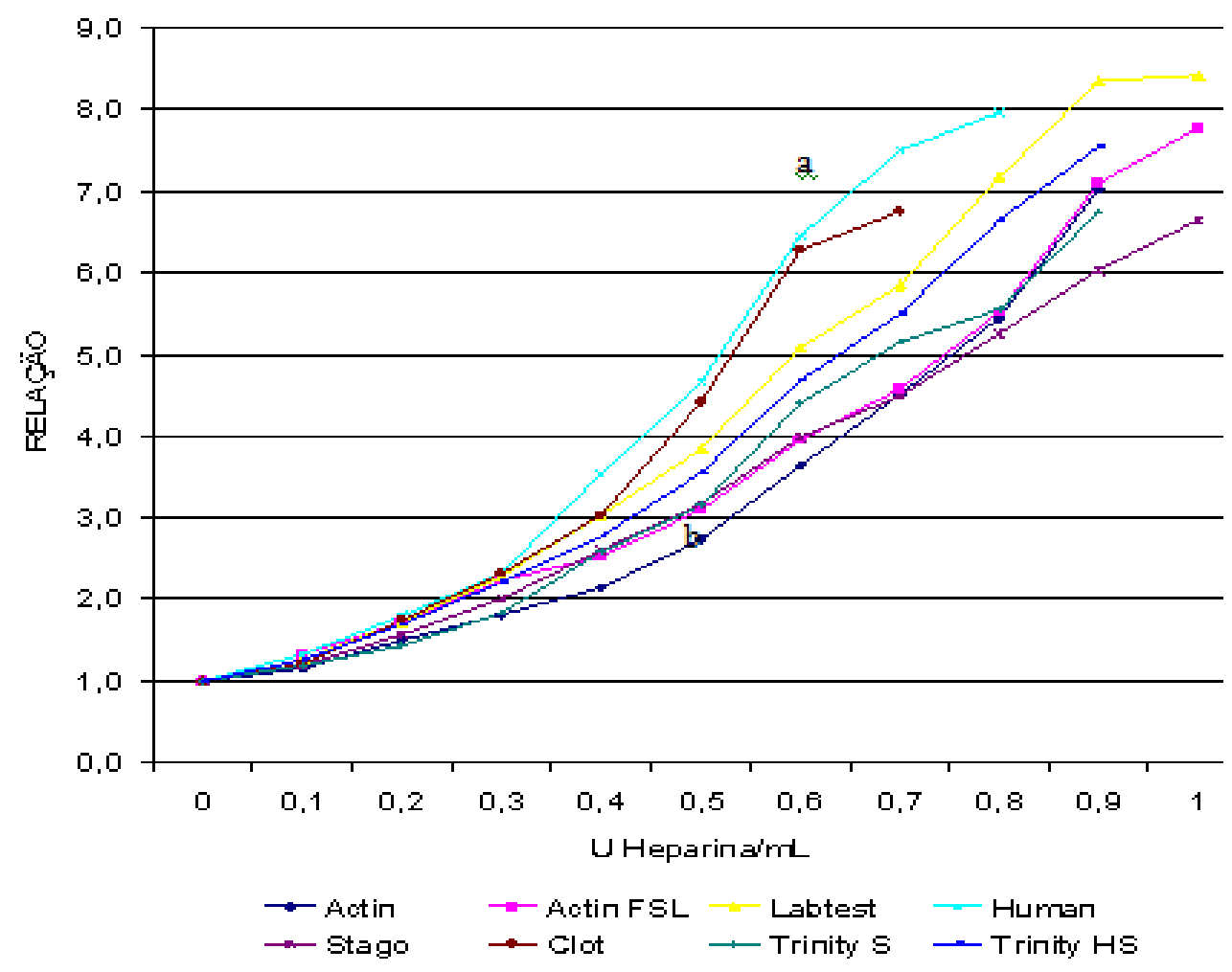

ACTIN - DADE-BEHRINGâ Actinâ; ACTIN FS - DADE-BEHRINGâ Actin® FS; HUMAN- HUMAN DO BRASIL ${ }^{\circ H E M O S T A T ~ A P T T-E L ; ~ S T A G O ~-~ D I A G N O S T I C A ~}$ STAGOÂSTA PTT A; TRINITY S - TRINITY BIOTECHâTriniCLOT aPTT S; TRINITY HS - TRINITY BIOTECHâTriniCLOT aPTT HS; CLOT - BIOS DIAGNÓSTICAâTTPA CLOT; LABTEST - LABTESTâ APTT Hemostasis

Fonte: autores

O reagente da Stago ${ }^{\circledR}$ apresentou o melhor resultado do TTPA versus concentração de HNF ( coeficiente de correlação $\left(\mathrm{R}^{2}=0,9919\right)$ para o Tabela 2). 
Tabela 2 - Valores do coeficiente de correlação (R2), resultados de Tempo de Tromboplastina Parcial Ativado (TTPA) versus a concentração de heparina $(0,1 ; 0,2 ; 0,3 ; 0,4 ; 0,5 ; 0,6 ; 0,7$; 0,$8 ; 0,9$ e 1,0 unidade de heparina/mL) em plasmas heparinizados in vitro

\begin{tabular}{|c|c|}
\hline MARCA/PRODUTO & $\mathbf{R}^{2}$ \\
\hline Dade-Behring $^{(\mathbb{B}} / \operatorname{Actin}^{(\mathbb{R}}$ & 0,9582 \\
\hline Dade-Behring ${ }^{\circledR} /$ Actin $^{\circledR}$ FSL & 0,9751 \\
\hline Labtest $^{\circledR} /$ APTT Hemostasis & 0,9868 \\
\hline Human do Brasil $^{\circledR} /$ HEMOSTAT aPTT-EL & 0,9800 \\
\hline Diagnostica Stago ${ }^{\circledR} /$ STA PTT A & 0,9919 \\
\hline Bios Diagnostica $^{\circledR} /$ TTPA CLOT & 0,9678 \\
\hline Trinity Biotech ${ }^{\circledR} /$ TriniCLOT aPTT S & 0,9807 \\
\hline Trinity Biotech $^{\circledR} /$ TriniCLOT aPTT HS & 0,9835 \\
\hline
\end{tabular}

Fonte: autores

Empregando plasmas de pacientes em uso de HNF os resultados do ACTIN e do ACTIN FSL foram estatisticamente diferentes dos da CLOT. Os resultados com reagentes Labtest ${ }^{\circledR}$ e Human ${ }^{\circledR}$ foram diferentes dos da STAGO e CLOT.

Já os valores obtidos com o reagente Stago $\AA$ foram diferentes daqueles com reagentes TRINITY S e os da CLOT foram diferentes daqueles com reagente TRINITY S e TRINITY HS (Figura 2).

Os reagentes Clot e Labtest apresentaram a maior e a menor média da $\mathrm{R}$, respectivamente (Figura 2). $\mathrm{Na}$ Tabela 3, está demonstrada a porcentagem dos pacientes por faixa de $\mathrm{R}$ dos diferentes kits utilizados.

Tabela 3 - Distribuição dos 29 pacientes em uso de heparina não-fracionada de acordo com os valores da relação (TTPA paciente/TTPA pool) e kits comerciais utilizados para a determinação do Tempo de Tromboplastina Parcial Ativado (TTPA).

\begin{tabular}{|c|c|c|c|}
\hline \multirow{2}{*}{ Marca/Produto } & \multicolumn{3}{|c|}{ Relação (TTPA paciente/TTPA pool) } \\
\hline & até 1,5 & 1,5 a 2,5 & Acima de 2,5 \\
\hline $\begin{array}{l}\text { DADE-BEHRING }{ }^{\circledast} \\
\text { Actin }{ }^{\circledR}\end{array}$ & $4(14 \%)$ & $21(72 \%)$ & $4(14 \%)$ \\
\hline $\begin{array}{l}\text { DADE-BEHRING }{ }^{\circledR} \\
\text { Actin® FS }\end{array}$ & $6(21 \%)$ & $19(66 \%)$ & $4(14 \%)$ \\
\hline $\begin{array}{l}\text { LABTEST }^{\circledast} \\
\text { APTT Hemostasis }\end{array}$ & $13(45 \%)$ & $16(55 \%)$ & $0(0 \%)$ \\
\hline $\begin{array}{l}\text { HUMAN DO BRASIL } \\
\text { HEMOSTAT aPTT-EL }\end{array}$ & $14(48 \%)$ & $14(48 \%)$ & $1(3 \%)$ \\
\hline $\begin{array}{l}\text { DIAGNOSTICA STAGO }{ }^{\circledR} \\
\text { STA PTT A }\end{array}$ & $2(7 \%)$ & $21(72 \%)$ & $6(21 \%)$ \\
\hline $\begin{array}{l}\text { BIOS DIAGNÓSTICA }{ }^{\circledast} \\
\text { TTPA CLOT }\end{array}$ & $1(3 \%)$ & $19(66 \%)$ & $9(31 \%)$ \\
\hline $\begin{array}{l}\text { TRINITY BIOTECH }^{\circledast} \\
\text { TriniCLOT aPTT S }\end{array}$ & $14(48 \%)$ & $12(41 \%)$ & $3(10 \%)$ \\
\hline $\begin{array}{l}\text { TRINITY BIOTECH } \\
\text { TriniCLOT APTT HS }\end{array}$ & $5(17 \%)$ & $20(69 \%)$ & $4(14 \%)$ \\
\hline
\end{tabular}

Os valores do teste de repetibilidade estão demonstrados na Tabela 4. O CV e o DP, em todos os reagentes, foram abaixo de 1,4 e 0,5 , para a amostra normal (Amostra A) e 2,1 e 1,5 para a amostra alterada (Amostra B), respectivamente. 
Figura 2 - Resultados de TTPA expressos em relação (TTPA paciente/TTPA pool de plasma), empregando oito kits comerciais e plasmas de pacientes em uso de heparina não-fracionada. a- Clot versus Actin, Actin FSL, Trinity S, Trynity HS, Labtest, Human; b- Stago versus Labtest, Human e Trinity S; c- Human versus Stago e Clot. Análise estatística realizada pelo Teste de Friedman, foi considerada significância estatística quando o $p<0,05$.

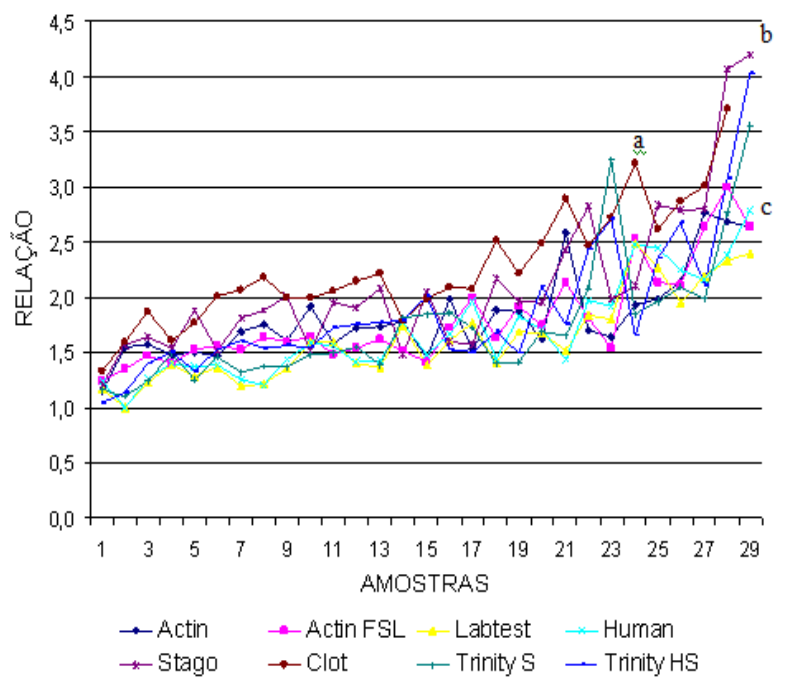

ACTIN - DADE-BEHRINGâ Actinâ; ACTIN FS DADE-BEHRINGâ Actin ${ }^{\circledR}$ FS; HUMAN- HUMAN DO BRASIL®HEMOSTAT APTT-EL; STAGO DIAGNOSTICA STAGOâSTA PTT A; TRINITY S - TRINITY BIOTECHâTriniCLOT aPTT S; TRINITY HS - TRINITY BIOTECHâTriniCLOT aPTT HS; CLOT - BIOS DIAGNÓSTICAâTTPA CLOT; LABTEST LABTESTâ APTT Hemostasis

Fonte: autores

\section{Discussão e Conclusão}

Vários fatores afetam a resposta do TTPA à heparina, incluindo: variáveis pré-analíticas, tais como o método de coleta e processamento da amostra biológica; variáveis analíticas, em especial a combinação do reagente e o instrumento utilizado para determinar o TTPA e variáveis biológicas, que incluem os níveis de fator de coagulação e aquelas que afetam a farmacocinética da heparina não fracionada (EIKELBOOM; HIRSH, 2006). As variáveis do paciente e do reagente para TTPA são as que apresentam maior influencia na variabilidade dos resultados do TTPA em pacientes heparinizados (OLSON, 2004).

A administração de heparina não fracionada por via intravenosa é amplamente utilizada para o tratamento do tromboembolismo venoso e o monitoramento terapêutico, como citado anteriormente, é obtido através do teste de TTPA (KITCHEN et al., 1996). A grande preocupação em relação ao TTPA decorre da diversidade de marcas de reagentes existentes no mercado, com diferentes composições e, conseqüentemente, diferentes sensibilidades à heparina (WINKLER; SHEPPARD; FANTZ, 2007). Esta diferença na sensibilidade à heparina leva a uma significativa variabilidade nos resultados de TTPA, que podem ter implicações na prescrição

Tabela 4 - Teste de repetitibilidade de diferentes marcas de tromboplastinas empregadas na realização do Tempo de Tromboplastina Pacial Ativado (TTPA).

\begin{tabular}{clcccccccc}
\hline \multirow{2}{*}{ Amostras } & & ACTIN & $\begin{array}{c}\text { ACTIN } \\
\text { FSL }\end{array}$ & HUMAN & STAGO & TRINITY TRINITY & CLOT & LABTEST \\
\hline \multirow{6}{*}{ A } & & & & S & HS & & \\
& MÉDIA (sg) & 27,5 & 31,8 & 30,5 & 33,8 & 31,9 & 33,8 & 35,0 & 30,0 \\
& DP (sg) & 0,3 & 0,4 & 0,2 & 0,2 & 0,2 & 0,2 & 0,5 & 0,2 \\
& CV (\%) & 1,1 & 1,4 & 0,6 & 0,7 & 0,5 & 0,6 & 1,3 & 0,7 \\
& MINIMO (sg) & 27,1 & 30,7 & 30,2 & 33,3 & 31,5 & 33,5 & 34,5 & 29,5 \\
& MAXIMO(sg) & 28,5 & 32,5 & 30,9 & 34,4 & 32,3 & 34,3 & 36,4 & 30,4 \\
& MAX - MIN & 1,4 & 1,8 & 0,7 & 1,1 & 0,8 & 0,8 & 1,9 & 0,9 \\
\hline \multirow{4}{*}{ B } & MÉDIA (sg) & 61,7 & 70,6 & 62,6 & 75,6 & 75,2 & 83,0 & 81,4 & 65,5 \\
& DP (sg) & 1,3 & 1,5 & 0,5 & 1,1 & 0,7 & 0,6 & 1,2 & 0,8 \\
& CV (\%) & 2,1 & 2,1 & 0,7 & 1,4 & 0,9 & 0,7 & 1,5 & 1,2 \\
& MINIMO (sg) & 59,3 & 65,9 & 61,6 & 74,3 & 73,9 & 82,1 & 79,7 & 64,2 \\
& MAXIMO(sg) & 65,2 & 72,5 & 63,4 & 79,0 & 76,3 & 84,1 & 84,1 & 67,2 \\
& MAX - MIN & 5,9 & 6,6 & 1,8 & 4,7 & 2,4 & 2,0 & 4,4 & 3,0 \\
\hline
\end{tabular}

ACTIN - DADE-BEHRINGâ Actinâ; ACTIN FS - DADE-BEHRINGâ Actinß ${ }^{\circledR}$ FS; HUMAN- HUMAN DO BRASIL ${ }^{\circledR} H E M O S T A T$ aPTT-EL; STAGO - DIAGNOSTICA STAGOâSTA PTT A; TRINITY S - TRINITY BIOTECHâTriniCLOT aPTT S; TRINITY HS - TRINITY BIOTECHâTriniCLOT aPTT HS; CLOT - BIOS DIAGNÓSTICAâTTPA CLOT; LABTEST - LABTESTâ APTT Hemostasis 
deste anticoagulante e que por sua vez podem causar complicações clínicas tais como hemorragia ou trombose (OLSON, 2004).

Eikelboom e Hirsh (2006) e Winkler, Sheppard e Fantz (2007) citaram em seus trabalhos que, ao contrário do tempo de protrombina (TP), que conta com o INR para diminuir a variabilidade nos resultados, para o TTPA não existe esta padronização. A proposta de entidades como o College of American Pathologists (Colégio Americano de Patologistas) é que os laboratórios clínicos determinem sua própria faixa terapêutica. Para isso, recomenda-se um grupo mínimo de 30 a 40 pacientes que receberam heparina. A correlação do TTPA deste paciente com um nível de heparina deve ser obtida com a titulação com a neutralização do sulfato de protamina ou ensaio de atividade do anti-fator Xa. Entretanto, os métodos utilizados na obtenção desta correlação são demorados, caros e não estão disponíveis em muitos laboratórios. Desta forma, na falta de um sistema de padronização para o monitoramento da HNF pelo TTPA a escolha do reagente é muito importante pois diferenças na sensibilidade dos reagentes foram demonstradas em vários estudos nas décadas de 80 e 90, como os de Bjornsson e Nash (1986) que utilizaram 7 kits de reagentes para o teste de TTPA, o de Kitchen et al. (1996), com 9 reagentes, e Kitchen et al. (1999) com 7 reagentes. Notou-se que existia uma grande variabilidade nos resultados tantos do teste com ex vivo como in vitro. Brill-Edwars et al. (1993) mostraram variação de sensibilidade à heparina em testes realizados com amostras de pacientes e utilizando reagentes da mesma procedência, porém com lotes diferentes.

Como na maioria dos laboratórios, o monitoramento da terapia com HNF ainda é realizado pelo TTPA, uma série de estudos demonstraram uma correlação entre heparinização inadequada e recorrência de trombose. Como conseqüência direta destas observações a maioria dos laboratórios adotou como intervalo terapêutico valores de relação entre 1,5 a 2,5 para o reagente do TTPA em uso, que corresponde a uma concentração de heparina de 0,2-0,4Unidades $/ \mathrm{mL}$ baseada nos valores por titulação com protamina ou inibição do fator Xa (EBY, 1997; KITCHEN, 2000).

Nelson (1999) mostrou que há diferenças na concentração de heparina quando a determinação é realizada pelos métodos de titulação com a protamina ou inibição do fator Xa. Já, Cuker et al. (2008) mostram que essa faixa terapêutica, em comparação com o fator anti-Xa, não tem uma boa concordância interlaboratorial. Essas observações sugerem que mais estudos devem ser realizados para comprovar a melhora da variabilidade entre os reagentes e métodos.

No presente trabalho foram estudadas 8 marcas de reagentes para a determinação do TTPA, com diferentes composições (tabela 1), utilizando plasmas de pacientes em uso de HNF e plasmas heparinizados in vitro. Nestes dois casos foram observadas diferenças significativas estatisticamente nos resultados de TTPA, as quais foram dependentes da marca de reagente utilizado.

O melhor coeficiente de correlação (R2), resultado do TTPA versus concentração de HNF, foi observado com $(R 2=0,9919)$, ou seja, o reagente da Stago ${ }^{\circledR}$ mostrou a melhor correlação entre os resultados do TTPA com as concentrações de heparina.

Considerando a faixa terapêutica na qual os pacientes em terapia com HNF foram classificados, foi observada uma variabilidade de acordo com a procedência do reagente. Para valores de R até 1,5, o kit da Clot $\AA$ apresentou o menor número de pacientes (1) enquanto que o maior número foi observado com os kits da Human ${ }^{\circledR}(14)$ e Trinity $S \circledR(14)$. Para valores de $\mathrm{R}$ entre 1,5 e 2,5 o menor e o maior número de pacientes foram observados com a marca Trinity $S \bowtie$ (12) e $\operatorname{Actin}{ }^{\circledR}(21)$ e $\operatorname{Stago}{ }^{\circledR}(21)$, respectivamente. Já para valores de $\mathrm{R}$ acima de 2,5 o menor e o maior número de pacientes foram observados com a marca Labtest ${ }^{\circledR}$ (0) e $\operatorname{Clot}{ }^{\circledR}$ (9), respectivamente. Estes resultados indicam que dependendo da procedência dos reagentes utilizados para a determinação do TTPA o paciente pode ser classificado em diferentes faixas terapêuticas e isto pode interferir na prescrição da HNF. 
A maior média da $\mathrm{R}$ foi obtida com o reagente da Clot $\AA(2,26)$ e a menor com o reagente da Labtest ${ }^{\circledR}$ $(1,60)$. Estas diferenças significativas nos resultados da $\mathrm{R}$ podem ser explicadas pela diferença na sensibilidade dos reagentes de marcas diversas em relação à HNF.

No teste de repetibilidade, os resultados obtidos mostram uma ótima performance dos reagentes, conforme indicado pelos seus fabricantes.

O presente estudo demonstrou que ainda existem diferenças estatísticamente significativas nos valores de TTPA em relação a sensibilidade a heparina de acordo com o kit comercial empregado para sua determinação.

\section{Referências}

BJORNSSON, T. D.; NASH, P. V. Variability in heparin sensitivity of APPT reagents. American Journal of Clinical Pathology. Philadelphia, v. 86, n. 2, p. 199-204, 1986.

BRILL-EDWARDS, P.; GINSBERG, J. S.; JOHNSTON, M.; HIRSH, J. Establishing a therapeutic range for heparin. Annals of Internal Medicine. Philadelphia v. 119, n. 2, p. 104-109, 1993.

CUKER, A.; PTASHKIN, B.; KONKLE, B. A.; PIPE, S. W.; WHINNA, H. C.; ZHENG, X. L.; CINES, D. B.; POLLAK, E. S. Interlaboratory agreement in the monitoring of unfractionated heparin using the anti-factor Xa-correlated activated partial thromboplastin time. Journal of Thrombosis and Haemostasis, Oxford, v.7, n. 1, p. 80-86, 2008.

EBY, C. Standardization of APTT Reagents for heparin therapy monitoring: Urgent or fading priority? Clinical Chemistry, Baltimore, v. 43, n. 7, p. 1105-1107, 1997.

EIKELBOOM,J.W.;HIRSH,J.Monitoringunfractionated heparin with the aPTT: time for a fresh look. Thrombosis and Haemostasis, Stuttgart, v. 96, n. 5, p. 547-552, 2006.

HAMERSCHLAK, N.; ROSENFELD, L. G. M. Utilização da heparina e dos anticoagulantes orais na prevenção e tratamento da trombose venosa profunda e da embolia pulmonar. Arquivos Brasileiro de Cardiologia, Rio de Janeiro, v. 67, n. 3, p. 209-213, 1996.
KITCHEN, S. Problems in laboratory monitoring of heparin dosage. British Journal of Haematology, Oxford, v. 111, n. 2, p.397-406, 2000.

KITCHEN, S.; CARTWRIGHT, I.; WOODS, T. A.; PRESTON, F. R. Lipid composition of seven APTT reagents in relation to heparin sensitivity. British Journal of Haematology, Oxford, v. 106, n. 3, p. 801-808, 1999.

KITCHEN, S.; JENNINGS, I.; WOODS, T. A. L.; PRESTON, F. E. Wide variability in the sensitivity of APPT reagents for monitoring of heparin dosage. Journal of Clinical Pathology. London, v. 49, n. 1, p. 10-14, 1996.

LIPPI, G.; FAVALORO, E. J. Activated partial thromboplastin time: new tricks for an old dogma. Seminars Thrombosis Hemostasis, New York, v. 34, p. 604-11, 2008.

NELSON, D. E. Current considerations in the use of the APTT in monitoring unfractionated heparin. Clinical Laboratory Science, Washington, v. 12, n. 6, p. 359-364, 1999.

OLSON, J. D. How to validate heparin sensitivity of the aPTT. 2004. Disponível em: <http://www.captodayonline. $\mathrm{com} /$ Archives/feature_stories/1004Heparin.html $>$. Acesso em: 31 jul. 2014.

POLLER, L.; THOMSON, J. M.; TABENER, D. A. Use of the activated partial thromboplastin time for monitoring heparin therapy: problems and possible solutions. $\mathrm{La}$ Ricerca in clinica e in Laboratorio, Milano, v. 19, n. 4, p. 363-370, 1989.

WINKLER, A. M.; SHEPPARD, C. A.; FANTZ, C. R. Laboratory monitoring of heparin: Challenges and opportunities. LabMedicine,. Chicago, v. 38, p. 499-502, 2007.

Recebido em: 31 jul. 2014. Aceito em: 14 fev. 2015. 